\title{
Trivium
}

Revue franco-allemande de sciences humaines et sociales - Deutsch-französische Zeitschrift für Geistesund Sozialwissenschaften

$19 \mid 2015$

Catégories de genre et mondes du travail

\section{Catégories de genre et mondes du travail}

Michel Lallement, Theresa Wobbe, Isabelle Berrebi-Hoffmann et Olivier Giraud

Traducteur : Isabelle Kalinowski

\section{OpenEdition}

\section{Journals}

Édition électronique

URL : http://journals.openedition.org/trivium/5044

ISSN : 1963-1820

\section{Éditeur}

Les éditions de la Maison des sciences de l'Homme

\section{Référence électronique}

Michel Lallement, Theresa Wobbe, Isabelle Berrebi-Hoffmann et Olivier Giraud, « Catégories de genre et mondes du travail », Trivium [En ligne], 19 | 2015, mis en ligne le 10 mars 2015, consulté le 08 septembre 2020. URL : http://journals.openedition.org/trivium/5044

Ce document a été généré automatiquement le 8 septembre 2020.

\section{(c) (i) (9)}

Les contenus des la revue Trivium sont mis à disposition selon les termes de la Licence Creative Commons Attribution - Pas d'Utilisation Commerciale - Pas de Modification 4.0 International. 


\title{
Catégories de genre et mondes du travail
}

\author{
Michel Lallement, Theresa Wobbe, Isabelle Berrebi-Hoffmann et Olivier \\ Giraud
}

Traduction : Isabelle Kalinowski

1 Quel est l'impact des catégories pour les relations entre les sexes ? Quel visage offre le monde du travail lorsqu'on l'examine dans cette perspective? Le présent numéro de Trivium éclaire la production de catégories et les modèles de classification à l'oeuvre dans le monde du travail et de la politique. Les contributions étudient l'ancrage institutionnel de ces catégories, ils analysent leurs capacités de transformation mais aussi de perpétuation et leurs répercussions éloignées. Ce numéro thématique est conçu comme une contribution aux débats sur l'histoire croisée et la socio-histoire (Historische Soziologie), qui prennent en compte les circonstances spécifiques de la production des catégories et l'espace dans lequel elles se sont développées. Il se propose d'apporter un complément à ce discours du point de vue de la sociologie du travail et de la sociologie du genre, qui constitue aussi le domaine de référence de notre programme de recherche franco-allemand intitulé « Les métamorphoses de l'égalité ${ }^{1}$ ».

2 La notion de catégorie au sens où nous l'entendons rassemble des attributs analogues entre lesquels des processus de classification permettent de tracer des frontières nettes. Comme le soulignent Emile Durkheim et Marcel Mauss, « il y a, au fond de notre conception de la classe, l'idée d'une circonscription aux contours arrêtés et définis ${ }^{2}$ ». Cette délimitation n'est pas de nature purement physique mais possède souvent une signification symbolique. Robert Hertz ${ }^{3}$, un jeune disciple de Durkheim, a pu ainsi contester le bien-fondé des interprétations « biologistes » d'une dissymétrie entre main droite et main gauche, dont on sait qu'elle hante encore nombre d'esprits contemporains. Sur les brisées du père de l'école française, Robert Hertz met en évidence l'association systématique dans notre conscience collective entre la droite, le sacré pur, l'homme, le courage, le haut, la rectitude ... d'un côté, et la gauche, le profane, le sacré impur, la lâcheté, le bas, la faiblesse ... d'un autre côté. Un raisonnement similaire peut s'appliquer au cas de la division du travail en fonction du 
genre, qui se fonde sur des partitions entre l'intérieur et l'extérieur, le travail domestique et le travail professionnel ${ }^{4}$. Les représentations symboliques sont avant tout construites autour d'oppositions binaires formées à l'aide de catégories mentales telles que, par exemple, haut / bas, masculin / féminin ou nature / culture ${ }^{5}$. Les représentations symboliques fonctionnent ensuite à l'aide d'analogies signifiantes qui engagent des ordres de réalité différents (biologiques, matériels, politiques, économiques ...) $)^{6}$. Mary Douglas montre plus explicitement encore que trois processus conjoints sont combinés dans les activités de classification : un travail de classification intellectuel basé sur la production de jugements de similitudes, un travail de classification sociale qui conduit ceux qui classent à se classer eux-mêmes (et à sélectionner par là-même leurs amis et leurs ennemis), un travail de mobilisation morale des membres de la société, enfin, qui contribue à la légitimation des classes et des identités. Comme le relève Isabelle Berrebi-Hoffmann ${ }^{7}$ en s'appuyant sur le cas des catégories du public et du privé, les sociologues ont tout intérêt à analyser les représentations à partir de configurations (comme la marchandisation de l'intime, la privatisation de la sphère publique...) qui permettent de mettre en évidence la pluralité des usages sociaux des catégories. Ainsi, par l'analogie entre masculin / féminin d'un côté, droite / gauche de l'autre, un principe social, celui de la division du travail, est conforté par une analogie physique.

3 Les processus d'attribution mutuelle d'une signification ne prennent forme qu'à partir de l'usage commun de catégories; ils aident les individus à comprendre leur environnement, donc eux-mêmes et d'autres personnes, mais aussi à ancrer des objets dans le monde social. Cette remarque s'applique de façon notable aux relations de genre. Le phénomène du genre lui-même est d'abord un phénomène de classification : une distinction est établie entre des personnes masculines et féminines, et la différence « entre deux sortes d'êtres humains » est ainsi « culturellement élevée à la conscience ${ }^{8}$ ».

4 Les acteurs, institutions et organisations du système de l'emploi sont dépendants de ces catégories. Dans son étude sur les carrières dans la police, Silvia Witz ${ }^{9}$ a montré que les classifications qui actualisent la différence interviennent moins au niveau formel que dans les pratiques quotidiennes. Lors de la mise en place de rondes de police, par exemple, l'idée selon laquelle les femmes sont moins à même de se défendre empêche de choisir des policières pour une patrouille collective. Dans leur étude sur le recrutement à des postes managériaux, Barbara Reskin et Debra Branch McBrier ${ }^{10}$ ont montré que, au niveau décisionnel des organisations, les attributs et les stéréotypes de genre s'imposent malgré eux aux décideurs dans le choix des candidats. Dans la mesure où la catégorisation par genre est ancrée dans des pratiques informelles d'organisation, notent ces auteures, elle résiste au changement, justement parce qu'elle est routinière et réduit la complexité sociale du processus d'organisation. De nombreuses études analysent les pratiques, institutions et discours qui produisent ces assignations et induisent la codification des activités professionnelles en fonction du genre.

5 Les modèles de signification ainsi produits peuvent rester attachés aux activités professionnelles sur un mode de relative inertie et, une fois stabilisés dans la durée, impliquer une partition du monde du travail en professions féminines et professions masculines qui n'admet en apparence pas d'alternative ${ }^{11}$.Dans le cas de l'industrie française, par exemple, Christian Baudelot et Michel Gollac ${ }^{12}$ ont montré que la reconnaissance des qualifications diffère selon le genre. Parce qu'elles sont réputées 
„naturellement“ patientes, les femmes qui effectuent des travaux qui impliquent de „ne pouvoir quitter son travail des yeux" ne bénéficient pas de primes de pénibilité équivalentes à celles des hommes qui effectuent des travaux de même nature. Leur salaire s'en trouve affecté d'autant.

6 Les études sur la catégorisation interactive des modèles de différence de genre dans les contextes professionnels ${ }^{13}$, sur les schémas de recrutement des organisations ${ }^{14}$ ou sur la puissance culturelle des stéréotypes pour la ségrégation des marchés du travail ${ }^{15}$ montrent que, aux différents niveaux de la société, cette catégorisation peut jouer le rôle d'un levier efficace dans l'accès à certaines positions. En résumé, les classifications n'existent pas seulement dans les têtes des individus et ne constituent pas seulement des horizons de référence de leur action : elles sont aussi confortées dans des structures symboliques et sociales.

7 La répartition des personnes en fonction de la différence de genre constitue certes un mode de classification extrêmement efficace, mais il n'est pas absolument nécessaire qu'elle induise aussi une différenciation sociale ${ }^{16}$; dans certains cas, elle peut même être abandonnée ou laissée en suspens ${ }^{17}$. Et même dans les cas où une répartition par classes de genre intervient ${ }^{18}$, la différenciation sociale associée à cette classification n'est pas nécessairement durable. Lorsque nous appréhendons des catégories comme socialement constituées, nous les concevons par là même comme des faits sociaux contingents.

8 Comme d'autres catégories du savoir ${ }^{19}$, celles du monde du travail sont riches de présupposés d'un point de vue social et elles ont une histoire. Compte tenu de leur historicité, elles peuvent aussi être entendues comme des objets historiques ${ }^{20}$ apparus le plus souvent dans le contexte de problèmes d'action et de controverses. Ainsi, dans la deuxième moitié du XIXe siècle, les règlementations sur le droit du travail et l'étude statistique du marché du travail représentent une tentative pour appréhender et ordonner sur un mode juridique et administratif un agrégat social jusque là inconnu qu'il va de soi pour nous aujourd'hui de désigner comme le marché du travail. La distinction entre les sexes n'a pas d'emblée été décisive dans ce contexte. Les statistiques professionnelles prussiennes répertorient, à partir de 1915, des individus masculins et féminins, sans associer à cette partition des assignations spécifiques ni des mises en garde contre des risques sociaux. Ce n'est qu'avec l'introduction, dans le règlement des métiers de 1878, de l'interdit de travail pour les accouchées dans les usines et pour les ouvrières de certaines branches que cette différence acquiert une pertinence, spécifiée dans l'amendement de $1891^{21}$.

9 Avec l'expertise médicale et les enquêtes des sciences sociales, cette codification encourage l'émergence des catégories d' " ouvrière » et de «travail des femmes ». Vers 1900, en Allemagne et en France (mais aussi dans d'autres pays), une représentation des «femmes» comme groupe spécifique et problématique sur le marché du travail, incarnant des risques sociaux, est associée à ces catégories ${ }^{22}$. Ces processus de classification vont également de pair avec des controverses nationales et internationales sur l'opportunité d'une législation dite de protection pour les individus féminins; elles portent sur la concurrence entre hommes et femmes, sur des thématiques de politique démographique et de politique familiale, mais aussi sur le risque social du chômage (surtout pour les individus masculins) ${ }^{23}$. Les discours sur «l'ouvrière » comme figure publique inquiétante et controversée font leur apparition 
en même temps que la catégorie du travail salarié médiatisé par un marché et, avec elle, celle de chômage ${ }^{24}$.

10 Au cours de cette période, au tournant du XXe siècle, Emile Durkheim, Georg Simmel, Werner Sombart et Max Weber ${ }^{25}$ s'intéressent aux conditions de travail et aux relations entre les sexes. Les catégories qui constituent la base de leur nouveau langage sociologique se fondent sur des oppositions. Elles correspondent aux représentations des époques passées mais aussi du présent, qui se transforme à une vitesse sidérante sous les yeux des sociologues, aussi bien dans le domaine économique que dans le domaine politique. La division du travail dans les rapports entre les sexes n'est pas seulement un thème clé chez Durkheim, mais aussi chez Simmel. Ce dernier regarde comme souhaitable une nouvelle répartition des professions dans laquelle les éléments "spécifiquement adaptés au mode d'activité féminin" seraient "réunis dans des professions particulières, différenciées ${ }^{26}$.

11 Aujourd'hui, les fondements structurels de ces classifications ne sont plus présents et les attentes formelles se sont reportées sur l'égalité. Pourtant, ces schémas peuvent encore être actualisés, comme le montrent les études sur les stéréotypes comme modèles de répartition légitime des professions dans des groupes de personnes et leurs effets de production d'inégalités ${ }^{27}$.

12 Pour mieux comprendre cette dynamique complexe, une contextualisation historique est précieuse. Une fois établies, en effet, les catégories semblent aller de soi et exclure toute alternative: leur caractère socialement construit demeure caché. Dans les processus de changement, il se donne cependant à voir, en révélant sa contingence. La relation entre réflexion sociologique et changement social illustre le même phénomène.

13 Lorsque, après 1945 , la sociologie des entreprises et de l'industrie et la sociologie $d u$ travail se constituent comme un nouveau domaine de spécialisation, elles se focalisent sur le travail industriel spécialisé. Les formes d'activité des femmes et des immigrés dans d'autres secteurs et segments du marché du travail ne retiennent guère l'attention ${ }^{28}$. Ce biais est ensuite remis en cause de différentes manières: avec les études féminines des années 1970 mais aussi avec les changements intervenus dans le système d'emploi, l'objet même de la sociologie du travail et de la sociologie de l'industrie se trouve déplacé. Elles s'intéressent aujourd'hui à la disparition du plein emploi industriel, remplacé par d'autres formes comme le temps partiel, les mini-jobs ou les « petits boulots » et laminé par l'impératif de flexibilitée ${ }^{2}$.

14 Les changements sociaux dans les rapports entre les sexes sont également en cause dans ce processus. Ainsi, la politique d'emploi européenne cherche à promouvoir l'employabilité des femmes tandis que la flexibilisation des conditions d'emploi, en Allemagne par exemple, induit des changements de fond dans le droit du travail et le droit social. La France n'échappe pas davantage à ces changements. Il est intéressant de noter par exemple combien la catégorie de temps partiel peut, dans ce pays, susciter des controverses, véhiculer des représentations souvent erronées pour contribuer, au nom de différenciations sexuées, à la promotion de nouvelles politiques du travail et de l'emploi tournées, comme en Allemagne, vers plus de flexibilité.

15 Dans l'Union Européenne, différents indices suggèrent l'émergence de nouveaux modèles d'accès aux revenus qui confèrent aux femmes des rôles inédits ; le modèle qui voulait que l'homme soit seul responsable de l'entretien du foyer est en recul et les couples où la femme est seule à avoir des revenus se multiplient. D'un autre côté, si des 
schémas anciens tel que les écarts de salaire entre hommes et femmes ont été quelque peu remis en cause ces dernières années en raison de la crise, il subsiste des différences importantes, de l'ordre de 16,4 \% en moyenne en Europe ${ }^{30}$.

16 Ces phénomènes renvoient à une configuration contrastée de nouvelles caractéristiques structurelles qui coexistent à côté de catégories anciennes de la sémantique des sexes; ces dernières sont cependant en recul dans les politiques de l'emploi et les politiques familiales. Les obstacles formels qui éloignaient auparavant les femmes du marché du travail sont aujourd'hui écartés. Mais ils ont fait place à des tendances indirectes et informelles qui, telle une main invisible, semblent répartir les individus masculins et féminins dans des champs professionnels distincts et contribuer à tracer des frontières sociales et symboliques.

17 Dans ce contexte, le présent numéro thématique de Trivium évalue le poids qui revient à certaines catégories et schèmes de classification. Nous avons choisi des contributions dont certaines portent sur de vastes périodes historiques (Yvonne Knibiehler, Theresa Wobbe). D'autres s'intéressent aux modes de catégorisation à l'intérieur du monde du travail (Danièle Kergoat, Bettina Heintz et Eva Nadai). Les deux dernières contributions discutent l'impact de la catégorie de genre dans le contexte politique (Laure Bereni et Eleanor Lepinard, Regina Gildemeister et Günther Robert).

18 L'article d'Yvonne Kniebiehler sur les médecins et la "nature féminine » est une étude pionnière et novatrice sur la genèse, autour de 1800 , des catégories de différenciation sexuée qui ont rempli, à la fin du XIXe siècle, la fonction d'un horizon d'attente social. Ce texte, qui offre en même temps des aperçus remarquables sur les sources françaises, met l'accent sur la continuité entre la phase pré-révolutionnaire et l'époque de Napoléon. L'auteure se demande dans un premier temps pourquoi l'essor des femmes qui semblait possible au début de la Révolution a connu si rapidement un coup d'arrêt. Pour expliquer ce retournement, Yvonne Kniebiehler remonte aux auteurs des Lumières, à leur invention de l'anthropologie et de l'histoire naturelle, qui font de l'homme un objet d'étude et d'expérience pratique. Comme l'a souligné Claudia Honegger dans L'Ordre des sexes (Die Ordnung der Geschlechter, 1991), ces sciences se fondent sur de nouveaux principes cognitifs : l'observation empirique comparative et une nouvelle vision de la corporéité.

19 Les médecins, note Yvonne Kniebiehler, tirent parti de cette nouvelle tendance. La réorganisation des hôpitaux français leur offre la possibilité unique de comparer régulièrement un grand nombre de malades et d'établir des statistiques. Parmi ces écrits médicaux se distingue l'œuvre de Pierre Roussel (1742-1802) Le système physique et moral de la femme qui, à l'instar de tous les systèmes philosophiques de médecine de son temps, présente un modèle spécifique de la féminité. Roussel réunit pour la première fois trois champs de savoir jusque là séparés, celui du naturaliste (la femme comme " femelle de l'homme »), le champ social avec l'idée de la femme comme épouse et mère (préfigurée par Rousseau), enfin celui du moraliste. Roussel associe la sexualité, le corps et l'âme de la femme avec le modèle d'une féminité naturelle, globale et séparée.

20 Entre 1770 et 1825 , une rupture avec la tradition se fait ainsi jour. La position subordonnée des femmes est désormais consacrée par la science et confortée par un nouveau système de référence universel ${ }^{31}$. La production de nouvelles catégories se développe alors sur un mode exponentiel (frigidité, virginité, maternité, pudeur, morbidité, nervosité, hystérie...). 
21 La contribution de Theresa Wobbe éclaire un autre contexte de savoir social, en l'occurrence les débuts des statistiques administratives des professions qui, à la fin du XIXe siècle, sont censées appréhender la société industrielle et la richesse de la nation. Les statisticiens cherchent à comprendre les dynamiques du marché du travail et de l'emploi, à traduire ces configurations sociales complexes sous une forme qui se prête à un traitement administratif et à fournir ainsi des repères pour la maîtrise des risques sociaux. Comme l'article d'Yvonne Kniebiehler, celui de Theresa Wobbe étudie de nouvelles approches de la catégorisation de la différence des sexes. A la différence de l'approche médicale et anthropologique, la statistique professionnelle permet d'appréhender le langage institutionnel de l'administration et de l'économie. Elle traduit la constitution de nouvelles attentes placées dans le «travail ».

22 L'imposition des classifications de la statistique des professions, affirme Theresa Wobbe, représente un tournant structurel pour la codification des professions et des activités salariées en fonction du genre. On assiste à la mise en place d'un schéma d'analyse institutionnel qui réduit la conception moderne du travail à une activité exercée à vie et médiatisée par un marché ; d'autres pratiques de travail se trouvent ainsi exclues ou disqualifiées comme ne correspondant pas «réellement» ou complètement à un « travail $»^{32}$.

23 Vers 1900 , cette classification se rattache pour une part au discours médical de la spécificité physique des individus féminins (voir l'article d'Yvonne Knibiehler) et pour une part à de nouveaux discours juridiques et à de nouveaux paradigmes des sciences sociales: la définition statistique du travail domestique comme non-travail, travail secondaire ou travail auxiliaire, le discours juridique relatif au « besoin de protection » des ouvrières, ou encore, dans les sciences sociales, la figure des risques sociaux associés au «travail des femmes". Avec les statistiques administratives, cette catégorisation des personnes est pour la première fois traduite dans une vision numérique d'ensemble, codifiée par sexe, de la population active et passive.

24 L'article de Danièle Kergoat marque une rupture dans l'histoire de la sociologie du travail en France. En formulant dès le titre la question " Ouvrier = ouvrière ? », ce texte a joué à un double égard un rôle fondateur. D’abord, il défend le principe de l'autonomie des pratiques masculines et féminines au sein d'un même groupe : la classe ouvrière. C'est un pas décisif qui va dans le sens d'un abandon de l'idée de la femme comme catégorie indirecte, médiatisée - parce que subordonnée à un monde dominé par la figure de l'ouvrier masculin qualifié et travaillant dans la grande industrie. Danièle Kergoat propose d'opérer de nouvelles distinctions entre travail et activité afin de donner aux relations entre femmes et hommes un sens qu'ils partagent dans l'univers du travail. Cette opposition a fait école et rencontré un écho important et fructueux dans les recherches actuelles en sociologie du travail, notamment les études sur les conditions de travail et les statuts d'activité qui observent encore aujourd'hui, dans la population française, un fossé entre les hommes et les femmes.

25 La contribution de Bettina Heintz et Eva Nadai a marqué elle aussi une césure dans la recherche en sociologie. Elle se rapporte au demeurant à un autre débat. Il ne s'agit plus de l'établissement de "l'ouvrière » comme figure spécifique et autonome et de l'usage de la catégorie de "genre » comme outil d'analyse. Les débats des années 1990 reflètent une autre réalité : dans les rapports de genre, la sociologie et le système d'emploi. D'un point de vue théorique, la notion de "sexe " comme catégorie de classification sociale générale est-elle encore pertinente compte tenu des changements 
qui ont affecté les rapports de genre? Est-elle encore en mesure de permettre une saisie empirique de ces derniers?

Les auteures de l'article contextualisent la différence de genre en se demandant à quelles conditions et dans quels contextes elle représente encore une différence et possède une pertinence dans la société d'aujourd'hui. Compte tenu de l'inclusion sociale croissante des femmes, notent-elles, les barrières formelles qui empêchaient autrefois directement l'accès au marché du travail (interdictions ou restrictions inscrites dans le droit du travail, structures du temps de travail, limitations associées au droit du couple et au droit de la famille) sont tombées. Les différences sociostructurelles se sont réduites dans les rapports de genre, tandis que d'autres variables ont pris de l'importance: situation sociale, âge, région, carrière, etc. Une désinstitutionnalisation des rapports de genre s'opère conjointement, un changement dans les mécanismes de reproduction de la différence des sexes: celle-ci n'est plus (re)produite par des critères d'admission formels mais par des mécanismes indirects et neutres en apparence.

27 Cette thèse de la contingence contextuelle de la différence de genre se fonde, d'un point de vue empirique, sur des études relatives aux professions dites «contrasexuelles », dans lesquelles hommes et femmes constituent des minorités (par exemple, les hommes dans les métiers infirmiers et les femmes dans l'informatique). Les auteures montrent comment la différence de genre est renforcée dans le quotidien des professions par des processus interactifs de marquage de frontières (boundary work).

28 Les derniers articles analysent les constructions et les classifications de la différence de genre à partir des exemples de discours français sur la " parité » et de la politique d'égalité des chances en Allemagne. Sous le titre «Les femmes ne sont pas une catégorie », Laure Bereni et Eléanor Lépinard discutent des initiatives de quotas dans le domaine de la représentation politique, qui ont rencontré un écho important en France avec la campagne pour la parité. Elles replacent celle-ci dans le contexte de la constitution, à l'échelle du droit européen, d'une norme juridique d'interdiction des discriminations et, au niveau des Nations Unies, de « la Convention sur l'élimination de toute forme de discrimination à l'égard des femmes» (CEDAW). Elles étudient les stratégies de légitimation à l'œuvre dans ce double cadre et leur traduction juridicoinstitutionnelle. L'accent est mis sur les conséquences paradoxales que la contradiction entre universalisme et différence, entre républicanisme et politique de l'égalité des chances entraîne du point de vue de la représentation politique. Cet article propose ainsi une analyse de l'inscription des catégories dans l'histoire contemporaine. Il illustre les allers et retours entre les catégories qui font partie d'un répertoire politique national et celles qui font l'objet de circulations internationales et sont susceptibles de circuler d'un enjeu politique à un autre ${ }^{33}$, par exemple, de la lutte contre la discrimination raciale ou de la réflexion sur les formes démocratiques vers l'égalité de genre. Ce texte explore ainsi de façon exemplaire les tensions entre les inscriptions historiques des catégories et leurs ancrages sociaux et spatiaux.

29 L'article de Regine Gildemeister et Günther Robert analyse les «ruses de la catégorisation » et porte sur les ambivalences de l'institutionnalisation du genre dans le contexte allemand, à partir de la politique d'égalité des chances. Les auteurs se demandent dans quelle mesure celle-ci s'appuie sur des constructions sociales qu'elle reformule elle-même par le biais de sa propre sémantique. Le constat de départ est que, au fur et à mesure qu'elle a gagné du terrain, la catégorie de "genre » a perdu le 
potentiel réflexif et critique qui était le sien (la différenciation des sexes ne précède pas le social, mais est le produit de ce dernier). D'un côté, la recherche d'un moyen terme entre l'universalisation et le singulier collectif restrictif «femme" induit des stéréotypisations de la différence de genre. D'un autre côté, le "genre » est devenu une catégorie d'usage courant en politique et dans l'administration: les acteurs parlent volontiers de constructions sociales mais, dans un même souffle, n'hésitent pas à prendre celles-ci pour point de départ des mesures qu'ils adoptent. Les ambivalences de l'institutionnalisation ne sont pas simplement imputables à des défaillances des acteurs individuels ni à un manque de professionnalisme des chargés de mission pour les politiques d'égalité des chances. Les ruses de la politique (d'égalité des chances), observent les auteurs de l'article, jouent à plein lorsqu'on cherche à supprimer les inégalités et, à cette fin, à parler au nom de toutes les «femmes ».

\section{BIBLIOGRAPHIE}

Amossé, Th. (2004) : « Professions au féminin. Représentation statistique, construction sociale », Travail, genre et sociétés, 1, 11, p. 31-46.

Angeloff, T. (2001) : «L'emploi féminin et le temps de travail : l'exemple du temps partiel », in : Groux, G. (ed.) : L'Action publique négociée. Approches à partir des 35 heures, France-Europe, Paris : L'Harmattan, p. 235-248.

Ayaß, W. (2000) : « “Der Übel größtes”. Das Verbot der Nachtarbeit von Arbeiterinnen in Deutschland (1891-1992), Zeitschrift für Sozialreform, 46, p. 189-220.

Baudelot, Ch. / Gollac, M. (1993) : « Salaires et conditions de travail), Economie et statistique, 265, p. 65-84.

Berrebi-Hoffmann, I. (dir.) (2009) : Politiques de l'intime. Des utopies sociales d'hier aux mondes du travail d'aujourd'hui, Paris : La Découverte.

Böhle, F. / Voß, G. / Wachtler, G. (éd.) (2010) : Handbuch Arbeitssoziologie. Lehrbuch, Wiesbaden : VS-Verlag.

Buscatto, M. / Marry, C. (2009) : « "Le plafond de verre dans tous ses éclats”. La féminisation des professions supérieures au XXe siècle », Sociologie du travail, 51 (2), p. 170-182.

Charles, M. / Bradley, K. (2009) : « Indulging Our Genderd Selves? Sex Segregetation by Field of Study in 44 Countries ", American Journal of Sociology, 114 (4), p. 24-76.

Cortéséro, R. / Kerbouc'h, S. / Mélo, D. / Poli, A. (2013) : « Recruteurs sous tensions. Discrimination et diversité au prisme de registres argumentaires enchevêtrés ", Sociologie du travail, 55 (4), p. 431-453.

Desrosières, A. (2005) : Die Politik der großen Zahlen. Eine Geschichte der statistischen Denkweise, Berlin : Springer.

Douglas, M. (1991 [1986]) : Wie Institutionen denken, trad. par Michael Bischoff, Francfort-sur-leMain : Suhrkamp. 
Durand, C. et al. (1985) : Le travail et sa sociologie. Essais critiques, Paris : L'Harmattan.

Durkheim, E. / Mauss, M. (1903) : « De quelques formes primitives de classification. Contribution à l'étude des représentations collectives ", texte extrait de L'Année sociologique, 6, p. 1-72. (Texte reproduit in Mauss, M. : Oeuvres. 2. Représentations collectives et diversité des civilisations, Paris : Les Éditions de Minuit, 1969, p. 13-89).

Fiske, S. T. (2005) : « What we know about the problem of the century: Lessons from social science to the law, and back », in : Nielsen, L. B. / Nelson, R. (éd.) : Handbook of employment discrimination research: Rights and realities, New York: Springer, p. 59-71.

Giraud, O. (2012) : «L'analyse scalaire des régimes d'action publique en Europe : l'apport méthodologique des comparaisons internationales ", Revue internationale de Politique Comparée, 19 (2), p. 15-36.

Glick, P / Fiske, S. T. (1999) : « Gender, Power Dynamics, and Social Interaction, in: Marx Ferree, M. / Lorber, J. / Hess, B. B. (éd.) : Revisioning Gender, Thousand Oaks, Calif.: Sage, p. 365-398.

Gildemeister, R. / Wetterer, A. (1992) : « Wie Geschlechter gemacht werden. Die soziale Konstruktion der Zweigeschlechtlichkeit und ihre Reifizierung in der Frauenforschung », in: Knapp, G.-A. / Wetterer, A. (éd.), TraditionenBrüche. Entwicklungen feministischer Theorie, Fribourg : Kore, p. 201-254.

Goffman, E. (1994 [1977]) : « Das Arrangement der Geschlechter », in : id. : Interaktion und Geschlecht. Francfort / New York : Campus, p. 105-158.

Hacking, I. (2004) : « Leute erfinden », in : id. : Historische Ontologie, trad. par Joachim Schulte, Zurich : Chronos, p. 119-134.

Heintz, B. / Nadai, E. (1998) : « Geschlecht und Kontext. De-Institutionalisierungsprozesse und geschlechtliche Differenzierung », Zeitschrift für Soziologie, 27, p. 75-93.

Hertz, R. (1970 [1928]) : Sociologie religieuse et folklore, Paris : PUF.

Hirschauer, S. (2014) : « Un/doing Differences. Die Kontingenz sozialer Zugehörigkeiten », Zeitschrift für Soziologie, 43 (3), p. 170-191.

Lamont, M. / Fournier, M. (éd.) (1992) : Cultivating Differences: Symbolic Boundaries and the Making of Inequality, Chicago : University of Chicago Press.

Maruani, M. / Meron, M. (2011) : Un siècle de travail des femmes en France, Paris: La Découverte.

Scott, J. W. (1994) : « Die Arbeiterin », in : Fraisse, G. / Perrot, M. (éd.) : Geschichte der Frauen. 19. Jahrhundert, t. 4., Francfort / New York : Campus, p. 451-480.

Renard, L. / Wobbe, T. / Matthieß, T. (2014) : «Statistisch unvergleichbar? Der Wandel der berufsstatistischen Kategorie "Mithelfende Familienangehörige" und die Metamorphosen der (west)deutschen geschlechterdifferenzierten Arbeitsordnung (1880-2010)», Working Paper ANRDFG Projekt “Metamorphosen der Gleichheit”. Geschlechtersoziologie - Universität Potsdam.

Reskin, B. F. / McBrier, D. B. (2000) : « Why not Ascription? Organisations' Employment of Male and Female Managers ", American Sociological Review, 65 (2), p. 210-233.

Ridgeway, C. L. (2001) : «Interaktion und die Hartnäckigkeit der Geschlechter-Ungleichheit in der Arbeitswelt », in : Heintz, B. (éd.) : Geschlechtersoziologie. Sonderheft 41 der Kölner Zeitschrift für Soziologie, Opladen, p. 250-275.

Ridgeway, C. (2011) : Framed by Gender. How Gender Inequality Persists in the Modern World, Oxford : Oxford University Press. 
Simmel, G. (1985 [1902]) : «Weibliche Kultur », Schriften zur Philosophie und Soziologie der Geschlechter, éd. par H.-J. Dahme et K. Ch. Köhnke, Francort-sur-le-Main : Suhrkamp, p. 159-176.

Somers, M. R. (1999) : « The Privatization of Citizenship: How to Unthink a Knowledge Culture », in : Bonnell, V. E. / Hunt, L. (éd.) : Beyond the Cultural Turn. New Directions in the Study of Society and Culture, Berkeley et al. : University of California Press, p. 121-164.

Tyrell, H. (2008 [1986]) : « Geschlechtliche Differenzierung und Geschlechterklassifikation », in : id. : Soziale und gesellschaftliche Differenzierung. Aufsätze zur soziologischen Theorie, Opladen : VS Verlag, p. 141-196.

Vanderstraeten, R. (2006) : « Soziale Beobachtungsraster: Eine wissenssoziologische Analyse statistischer Klassifikationsschemata », Zeitschrift für Soziologie, 35, p. 193-211.

Wadauer, S. (2014) : « Production of Work », Institut für Wirtschafts- und Sozialgeschichte, Universität Wien, http://pow.univie.ac.at/aktivitaeten/aktuelle-publikationen/; dernière visite le $12 / 12 / 2014$.

Wikander, U. / Kessler-Harris, A. / Lewis, J. (éd.) (1995) : Protecting Women: Labor Legislation in Europe, the United States, and Australia, 1880-1920, Campaign : University of Illinois Press.

Wilz, S. M. (2005) : « "Nicht genügend kann davor gewarnt werden ..." - Männer und Frauen bei der Polizei: Fakten und Diskurseé », in: Ahrens, J.-R. / Apelt, M. / Bender, C. (éd.) : Frauen im Militär. Empirische Befunde und Perspektiven zur Integration von Frauen in die Streitkräfte, Wiesbaden: VS Verlag für Sozialwissenschaften, p. 156-172.

Willms, A. (1980) : Die Entwicklung der Frauenerwerbstätigkeit im Deutschen Reich. Eine historischsoziologische Studie, Nuremberg: IAB.

Wobbe, T. (2014) : « Gleichbehandlung und Individualrechte: Das transnationale Geschlechterkonzept der EU aus historisch-soziologischer Sicht ", in : Heidenreich, M. (éd.) : Krise der europäischen Vergesellschaftung? Soziologische Perspektiven, Wiesbaden : Springer, p. 229-251.

Wobbe, T. / Berrebi-Hoffmann, I. / Lallement, M. (éd.) (2011) : Die gesellschaftliche Verortung des Geschlechts. Diskurse der Differenz in der deutschen und französischen Soziologie um 1900, Francfort / New York : Campus.

\section{NOTES}

1. « Métamorphoses de l'égalité II : modèles d'interprétation du genre à partir de l'exemple de la classification des statistiques professionnelles, des débats sur l'égalité des chances et des politiques d'égalité des chances dans le contexte franco-allemand (1945-2010) », Université de Postdam / CNRS Paris 2012-2015. Nous remercions la DFG et l'ANR d'avoir financé ce programme.

2. Durkheim / Mauss (1903), p. 2-3.

3. Hertz (1970 [1928]).

4. Collins (1992); Goffman (1977).

5. Cf. Lamont / Fournier (1992).

6. Douglas (1991 [1986]).

7. Berrebi-Hoffmann (2009).

8. Tyrell (2008 [1986]), p. 144 ; Goffman (1977).

9. Witz (2005).

10. Reskin / McBrier (2000).

11. Gildemeister / Wetterer (1992); Heintz / Nadai (1998); Maruani / Meron (2011); Reskin / McBrier (2000). 
12. Baudelot / Gollac (1993).

13. Amossé (2004) ; Buscatto / Marry (2009) ; Ridgeway (1997, 2011).

14. Cortéséro / Kerbouc'h / Mélo / Poli (2013).

15. Charles / Bradley (2009).

16. Heintz / Nadai (1998).

17. Hirschauer (2014).

18. Goffman (1977).

19. Cf. Durkheim / Mauss (1987).

20. Somers (1999).

21. Cf. Аyaß (2000).

22. Cf. Scott (1994).

23. Cf. Wikander et al. (1995).

24. Zimmermann (2006).

25. Cf. Wobbe / Berrebi-Hoffmann / Lallement (2011).

26. Dans certains syndicats anglais assurant un salaire standard pour hommes et femmes, le principe de la division du travail s'impose en l'absence même de concurrence salariale, note Simmel : «La division du travail s'était forgée d'elle-même : les femmes avaient pour ainsi dire monopolisé à leur profit les fonctions adéquates à leurs forces physiques et à leur habileté, laissant aux hommes celles qui convenaient à leurs forces" (Simmel [1902], p. 164). Simmel décrivait ainsi le mécanisme de différenciation sexuée à l'œuvre dans la division du travail, qui s'est conforté au cours du XXe siècle par le biais de la ségrégation en vigueur sur le marché du travail.

27. Cf. Fiske (2005) ; Glick / Fiske (1999) ; Ridgeway (2011).

28. Durand et al. (1985).

29. Angeloff (2001) ; Böhle / Voß / Wachtler (2010).

30. Cf. Wobbe (2014).

31. Cf. Hausen (1976); Honegger (1991).

32. A partir des "membres de la famille " qui «fournissent une aide» et forment un groupe intermédiaire entre travail et non-travail, entre famille et profession, on peut reconstituer le mécanisme de l'exclusion et de l'inclusion (Giraud [2012]; Renard / Wobbe / Matthieß [2014]). Les études menées dans le cadre du projet de recherche «The Production of Work » (Wadauer [2014]) à l'université de Vienne analysent ces processus d'exclusion et d'inclusion dans la perspective du travail exclu, à partir de l'exemple d'activités anciennes comme le vagabondage, le colportage, etc., vers 1900.

33. Giraud (2012).

INDEX

Schlüsselwörter : Arbeit, Geschlecht, Einleitung

Mots-clés : travail, genre, introduction 


\section{AUTEURS}

\section{MICHEL LALLEMENT}

Michel Lallement est professeur du Cnam titulaire de la chaire d'analyse sociologique du travail. Pour plus d'informations, voir le lien suivant.

\section{THERESA WOBBE}

Theresa Wobbe est professeure de sociologie à l'Université de Postdam. Pour plus d'informations, voir le lien suivant.

\section{ISABELLE BERREBI-HOFFMANN}

Isabelle Berrebi-Hoffmann est chargée de recherche CNRS au Laboratoire interdisciplinaire pour la sociologie économique du Conservatoire national des arts et métiers. Pour plus d'informations, voir le lien suivant.

\section{OLIVIER GIRAUD}

Olivier Giraud est Olivier Giraud est chargé de recherche CNRS au Laboratoire interdisciplinaire pour la sociologie économique du Conservatoire national des arts et métiers. Pour plus d'informations, voir le lien suivant. 